\title{
Partial Volume Correction of Small Animal PET Cardiac Dynamic Images Using Iterative Reconstruction: Effects on Glucose Metabolic Rate Measurement
}

\author{
D D’Ambrosio ${ }^{1,2}$, G Fiacchi $^{2,4}$, P Cilibrizzi $^{2}$, C Lamberti $^{4}$, G Baldazzi $^{3}$, \\ $\mathrm{S} \mathrm{Boschi}^{5}, \mathrm{R}_{\text {Franchi }}{ }^{5}, \mathrm{M}$ Marengo $^{2}$, A E Spinelli ${ }^{1,2}$ \\ ${ }^{1}$ Scuola di Specializzazione in Fisica Sanitaria, Universita' di Bologna, Italy \\ ${ }^{2}$ Servizio di Fisica Sanitaria, Policlinico S. Orsola - Malpighi, Bologna, Italy \\ ${ }^{3}$ Dipartimento di Fisica,Universita' di Bologna, Italy \\ ${ }^{4}$ DEIS, Universita' di Bologna, Italy \\ ${ }^{5}$ Servizio di Medicina Nucleare, Policlinico S. Orsola - Malpighi, Bologna, Italy
}

\begin{abstract}
Small animal positron emission tomography (PET) image quantitation is severely affected by partial volume effect (PVE) caused by the spatial resolution of PET tomographs. The aim of this work was to reduce the PVE using a modified iterative expectation maximization (EM) reconstruction algorithm. The goal of the method was to increase the accuracy of tracer concentration values in order to obtain an in vivo better estimate of physiological parameters. The performance of the proposed correction method was evaluated by calculating left ventricle and myocardium mean value using simulated cardiac fluorodeoxyglucose (FDG) dynamic images.
\end{abstract}

\section{Introduction}

Positron emission tomography (PET) images provide important functional information, however the poor spatial resolution typical of PET images can lead to partial volume effect (PVE). The net result of PVE is an incorrect measurement of the true radiotracer concentration.

As outlined by Aston et al. [1] PVE can be divided into two effects: tissue-fraction and point-spread effect. The tissue fraction effect arises from tissue heterogeneity because the region of interest (ROI) used to determine radiotracer concentration contains signals from different tissues (for example myocardium and left ventricle). The pointspread effect arises from the finite resolution of PET scanner. Correction for PVE is of great importance for both semi quantitative and quantitative measurements. Quantitative and more advanced approaches such as compartmental analysis (CA) are applied to measure the exchange of substances between several compartments within the hu- man body. CA is based on the measurement of the arterial input function (IF) and the tissue time activity curve (TAC). Typically the IF and TAC measurements are obtained from the image by drawing a set of ROIs and, thus, PVE can lead to large errors in the estimated kinetic rate constants. PVE can be corrected using several approaches see for example Bouvat et al for a review [2].

In this work a correction scheme based on an iterative image reconstruction approach including the modeling of the system point spread function (PSF) has been implemented. The paper is organized as follow: in section 2 the mathematical formalism for image reconstruction and simulations is introduced, in section 3 the main results are presented, discussion and conclusions then follow.

\section{Methods}

\subsection{Partial volume correction}

The basic equation of the expectation maximization (EM) algorithm [3] is :

$$
n_{j}^{k+1}=\frac{n_{j}^{k}}{\sum_{i=1}^{I} a_{i j}}\left[\sum_{i=1}^{I} a_{i j} \cdot \frac{m_{i}}{q_{i}^{k}}\right]
$$

with $q_{i}^{k}=\sum_{j=1}^{J} a_{i j} n_{j}^{k}$, where $q_{i}^{k}$ represents the estimated projection of the image along the $i$-th Line Of Response (LOR), $n_{j}^{k}$ represents the reconstructed image at the $k$-th step, while $n_{j}^{k+1}$ is the reconstructed image at the next iteration; $a_{i j}$ is an element of the system matrix $A$, and represents the probability that an emission from voxel $j$ will be detected along LOR $i ; m_{i}$ is the measured projection 
of the image along the LOR $i$. $I$ is the number of LORs while $J$ is the number of pixels of the image. The system point spread function can be introduced in the reconstruction step by modeling the scanner resolution in the system matrix [4]. More precisely the system matrix can be factorized as $A=P X$ where $\mathrm{X}$ is the radon transform and the matrix $P$ describes the system blurring in the projection space. The projection at the $k+1-t h$ step can be written as $q^{k+1}=P X n^{k}$.

In order to model accurately the system resolution the PSF was measured using a small $(0.2 \mathrm{~mm}) 18-\mathrm{F}$ point source [5]. The source was placed at the center of the field of view (FOV) and several measurements were taken by moving the source along the $y$ axis with an increment of $2 \mathrm{~mm}$. The resulting PSF (obtained from the sinogram) was then fitted using a Gaussian function in order to obtained the Full Width Half Maximum (FWHM) at different positions.

The spatially variant PSF was modeled in the forward step of the EM algorithm, more precisely the sinogram was blurred pixel by pixel accordingly to the following equation: $q_{i}^{k}=\sum q_{l}^{k} P S F_{i, l}$ [6].

\subsection{Image simulations}

In order to validate the proposed PVE correction method a set of simulations was performed to compare the glucose metabolic rate (GMR) before and after correction. More precisely a two compartments model was simulated considering an input function $C_{p}(t)$ and a set of kinetic rate constants $\left(K_{1} \ldots k_{4}\right)$ typical of an FDG scan. The tracer uptake $C_{t}(t)$ in the myocardium was obtained using the following expression:

$$
C_{t}(t)=\left(p_{1} e^{-p_{2} t}+p_{3} e^{-p_{4} t} \otimes\right) C_{p}(t)
$$

where the coefficients $p_{1} \ldots p_{4}$ are equal to:

$$
\begin{gathered}
p_{1}=\frac{K_{1}}{\alpha_{2}-\alpha_{1}}\left(k_{3}+k_{4}-\alpha_{1}\right) \\
p_{2}=\alpha_{1} \\
p_{3}=\frac{K_{1}}{\alpha_{2}-\alpha_{1}}\left(\alpha_{2}-k_{3}-k_{4}\right) \\
p_{4}=\alpha_{2} \\
\alpha_{1,2}=\left[\left(k_{2}+k_{3}+k_{4}\right) \pm \sqrt{\left(k_{2}+k_{3}+k_{4}\right)^{2}-k_{3} k_{4}}\right]
\end{gathered}
$$

In order to apply the Patlak graphical analysis the value of $k_{4}$ was set to 0 . The input function $C_{p}(t)$ was equal to [7]:

$$
C_{p}(t)=\left(A_{1} \cdot t-A_{2}-A_{3}\right) e^{\lambda_{1} t}+A_{2} e^{\lambda_{2} t}+A_{2} e^{\lambda_{3} t}
$$

where the coefficients $A_{1} \ldots A_{3}$ and $\lambda_{1} \ldots \lambda_{3}$ are chosen to mimic a mouse FDG input function. The theoretical GMR value was $7.7 \cdot 10^{-4} s^{-1}$. The simulated input fuction and myocardium time activity curve are showed in figure 1.
In order to obtain a dynamic cardiac image, curves with Poisson noise were applied to the heart of digital mouse phantom MOBY [8], then, the images were smoothed accordingly to the system PSF. In figure 2 some frames of the simulated dynamic image are shown.

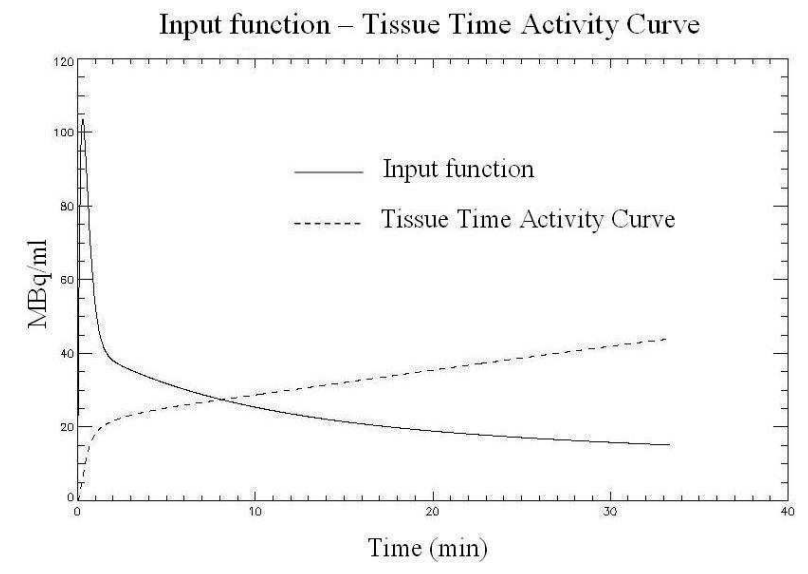

Figure 1. The figure show the simulated input function (full line) and the FDG myocardium time activity curve (dotted line).

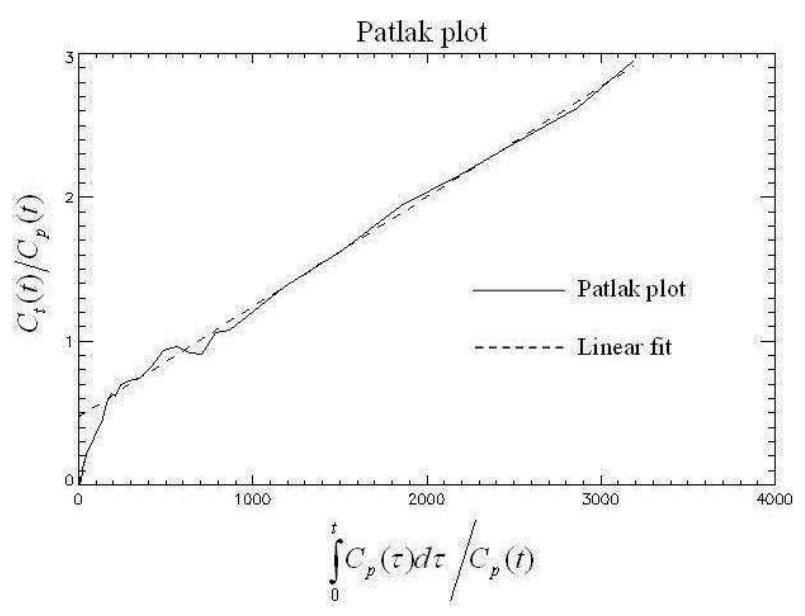

Figure 2. The figure show the Patlak plot and the linear fit from which the GMR was extracted as explained in section 2.2 .

For each pixel of the myocardium of both pre and post-correction images Patlak graphical analysis was performed. Time activity curve for each pixel of the myocardium was extracted from the corrected and uncorrected images and the pixel GMR $k_{m}$ was estimated as the angular coefficient of the Patlak plot accordingly to the following equation:

$$
\frac{C_{t}(t)}{C_{p}(t)}=\frac{K_{1} k_{2}}{\left(k_{2}+k_{3}\right)^{2}}+\frac{K_{1} k_{3}}{\left(k_{2}+k_{3}\right)} \frac{\int_{0}^{t} C_{p}(\tau) d \tau}{C_{p}(t)}
$$



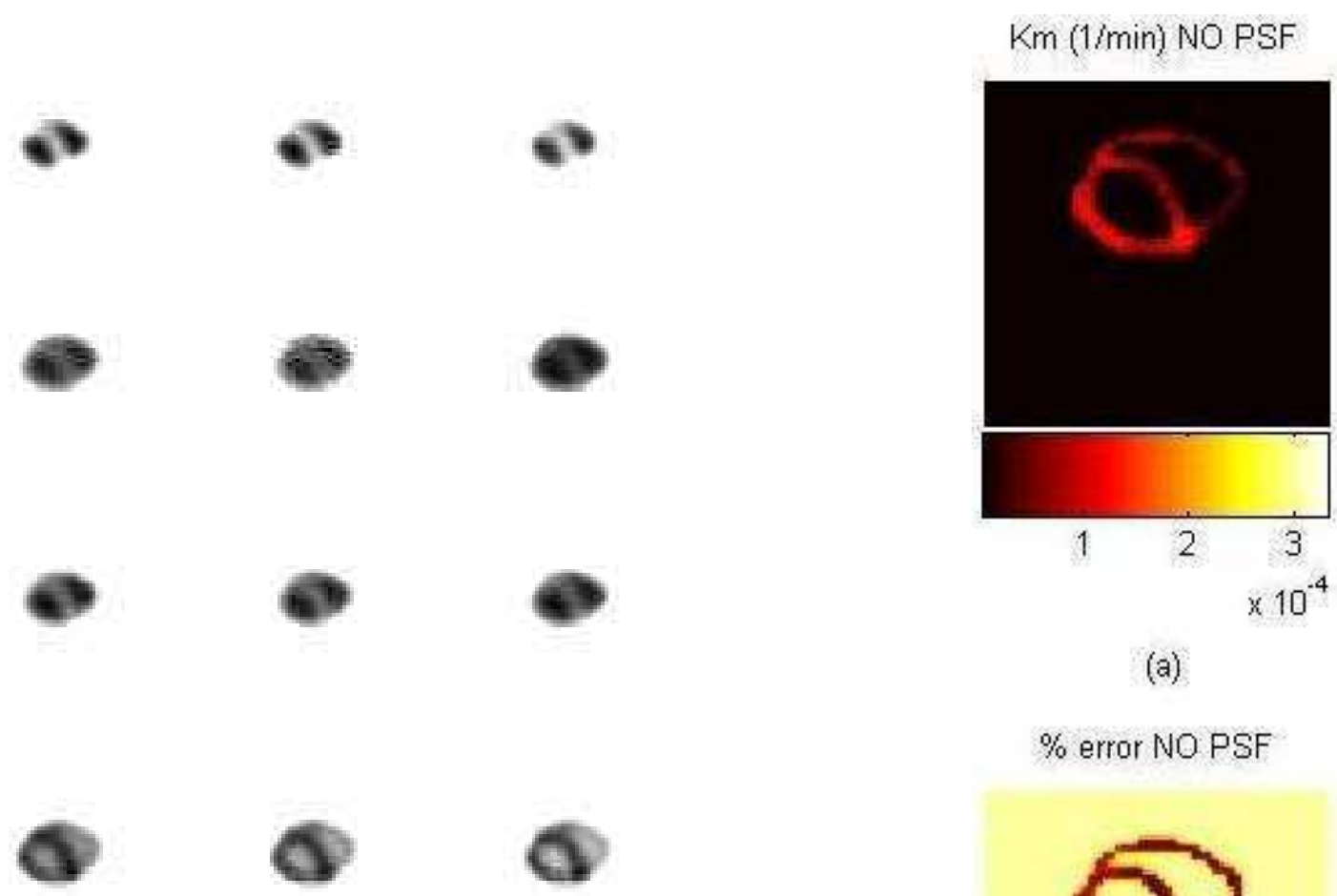

Figure 3. The figure show some representative frames of the simulated dynamic image of MOBY's heart.

where:

$$
k_{m}=\frac{K_{1} k_{3}}{\left(k_{2}+k_{3}\right)}
$$

Only the final part of the Patlak plot was included in the linear fit.

\section{Results}

In this section results obtained using EM reconstruction algorithm with and without PSF modeling are presented.

Figure 4 shows the GMR parametric image reconstructed without PSF modeling and the image including the errors with respect to the true GMR value $\left(k_{m}\right)$. Figure 5 show the parametric image reconstructed using EM with PSF modeling and the relative error map. The colorbar under the parametric images put in evidence the differences between uncorrected and corrected images.

In figure 6 are showed the input function extracted from images relative to the FDG simulation for the two compartmental model. The IF value is calculated as the mean value over the left ventricle. As one can see from the plot, the value of the input function peak is considerably grater in PVC curve respect to the non corrected one. The differences between the measured peak values are about $10 \%$ (a)

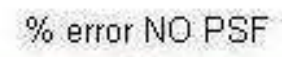

a)

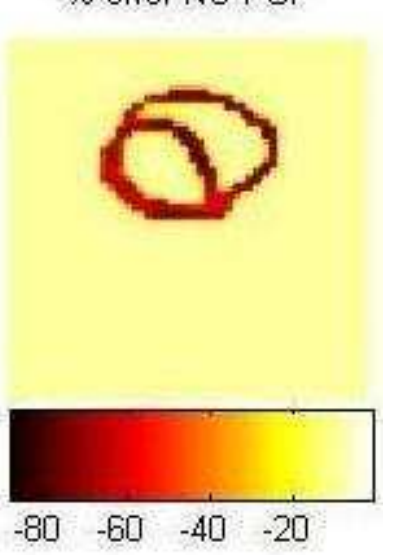

(b)

Figure 4. Parametric image of metabolic rate (a) and relative error respect to the teorethical $k_{m}$ value (b) for reconstruction algorithm without PSF modeling

and $40 \%$ for corrected and uncorrected images, respectively.

\section{Discussion and conclusions}

Pre and post-correction parametric images of metabolic rate and the relative errors show how the image reconstruction based PVC reduces errors with respect to the theoretical $k_{m}$ value in each pixel.

In particular, the error map of PVC parametric image show that the error on GMR estimation is greater on the edges of myocardium while the error is very low inside 


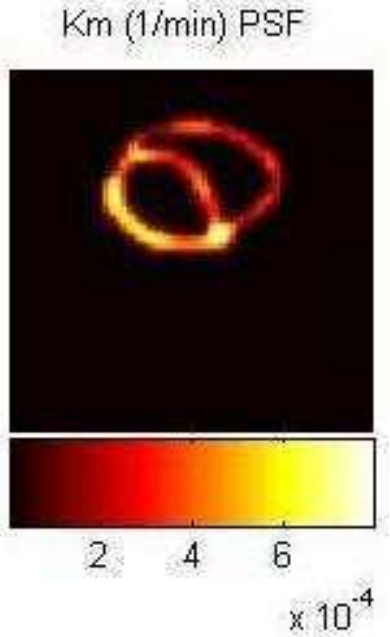

(a)

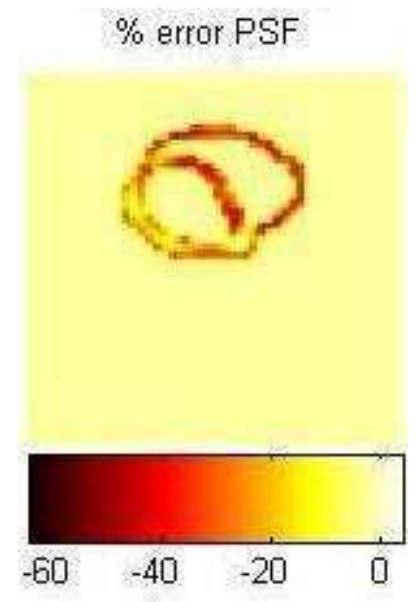

(b)

Figure 5. Parametric image of metabolic rate (a) and relative error respect to the teorethical $k_{m}$ value (b) for reconstruction algorithm with PSF modeling

the myocardium tissue, (see the left bottom corner of the heart). In general, PVE is more significant for thin structures, this explains why the error in the top right corner of the myocardium is greater. In conclusion, our findings show that iterative reconstruction with PSF modeling allow to obtain a better measure of the radiotracer concentration in the heart and, consequently, a more accurate estimation of input function and glucose metabolic rate.

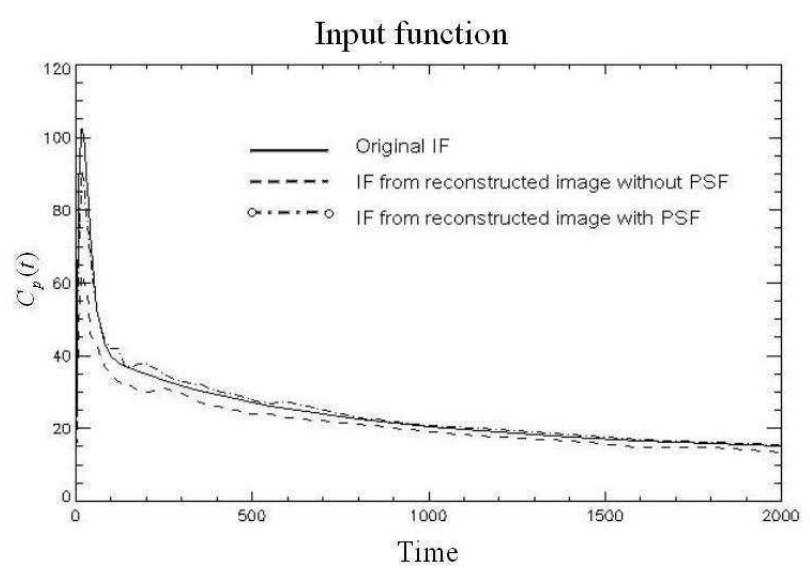

Figure 6. Comparison between input function measured on reconstructed images with and without PSF modeling. The continuous line represent the true IF.

\section{Acknowledgements}

The authors would like to acknowledge the Fondazione del Monte di Bologna for the financial support.

\section{References}

[1] Aston J, Cunningham V, Asselin MC, Hammers A, Evans A, Gunn R. Partial volume correction: Estimation and algorithms. J. Cereb. Blood Flow Metab., 2002; 22:1019-34

[2] Soret M, Bacharach S L, Buvat I. Partial-Volume effect in PET tumor imaging. J. Nucl. Med., 2007; 48:932-45

[3] Shepp LA, Vardi Y. Maximum Likelihood Reconstruction for Emission Tomography. IEEE Trans. Nucl. Sci., 1982; MI-1.

[4] Reader AJ, Julyan PJ,Williams H,Hastings DL, Zweit J. EM Algorithm System Modeling by Image-Space Techniques for PET Reconstruction. IEEE Trans. Nucl. Sci., 2003; 50.

[5] Spinelli AE, D’Ambrosio D, Pettinato C, Trepidi S, Nanni C, Ambrosini V, Baldazzi G, Bergamini C, Marengo M Performance evaluation of a Small Animal PET Scanner. Spatial resolution characterization using $18 \mathrm{~F}$ and $11 \mathrm{C}$ Nucl. Instr. and Meth. in Phys. Res. A, 2007; 571:215-8

[6] Brix G,Doll J,Bellemann ME,Trojan H,Haberkorn U, Shmidlin, Ostertag H. Use of scanner characteristics in iterative image reconstruction for high-resolution positron emission tomography studies of small animals. Eur J. Nucl. Med., 1997; 24:779-86.

[7] Feng D, Huang SC, Wang X. Models for computer simulations studies of input functions for tracer kinetic modeling with positron emission tomography. Int. J. Biomed. Comput., 1993; 32:95-110

[8] Segars WP, Tsui BMW, Frey EC, Johnson GA, Berr SS. Development of a 4D digital mouse phantom for molecular imaging research. Molecular Imaging and Biology, 2004; 6(3):149-59.

Address for correspondence:

Antonello E Spinelli, Ph.D.

Servizio di Fisica Sanitaria,

Policlinico S. Orsola - Malpighi,

Via Massarenti, N. 9,

40138, Bologna, Italy

E-mail address: antonello.spinelli@unibo.it 